\title{
Motion mode and two-dimensional echocardiographic measurements of cardiac dimensions in local dogs in Malaysia
}

\begin{abstract}
Echocardiographic measurements of the dog's heart are known to be breed-specific. The establishment of normal echocardiographic measurements for each breed of dog increases the accuracy of echocardiographic examination. The purpose of this preliminary study was to determine the value of normal echocardiographic measurements of local dogs from Malaysia. A total of 20 clinically healthy client-owned pet dogs were recruited, in accordance to the predetermined inclusion criteria of the study. Two dimensional and M-mode echocardiograms image of both long and short axis of the left ventricle were obtained in accordance to recommendation by the American Society of Echocardiography. From this study, the reference echocardiographic measurements of local dogs in Malaysia were established. The measurements of intra-cardiac dimensions, wall thickness, and contractility varied from the Indonesian mongrel dogs, as well as the general population of dogs that have the same average body weight. There is no association between echocardiographic measurements with gender and bodyweight of local dogs in Malaysia.
\end{abstract}

Keyword: Malaysia; Local dogs; Breed specific; Echocardiography; Reference measurements; Heart 